\title{
DER HANDELSRECHTLICHE UND STEUERRECHTLICHE GEWINNBEGRIFF IN DER BUNDESREPUBLIK DEUTSCHLAND
}

\author{
von Rudolf Thiel
}

\section{Allgemeiner Teil}

\section{Der dreifache Einkommensbegriff}

Diese Beitragsfolge hat sich zum Ziele gesetzt, den Begriff des Einkommens zu beschreiben, so wie er in den verschiedenen Ländern

a) von den Wirtschaftlern,

b) von den Wirtschaftsprüfern und

c) von den Steuerexperten

verstanden wird. $\mathrm{Da}$ sich die Wirtschaftler und Wirtschaftsprüfer vornehmlich mit dem Einkommen aus Handel und Gewerbe zu befassen pflegen, so wird sich dieser Beitrag vornehmlich mit jenem Einkommen zu befassen haben, wie es von den Kaufleuten, d.h. von den Handel- und Gewerbetreibenden aller Art, und von den Kapitalgesellschaften erwirtschaftet wird; dagegen wird das Einkommen, wie es z.B. von den Arbeitnehmern, Vermietern und privaten Kapitalbesitzern erzielt wird, außer Betracht bleiben dürfen. Nach der Konzeption, wie sie von Wisselink in seinem einleitenden Beitrag entworfen worden ist, soll bei der Darstellung des so eingeengten Einkommensbegriffs das Augenmerk besonders auch darauf gerichtet werden, inwieweit der Handelsbrauch, d.h. die Grundsätze, wie sie im Geschäftsverkehr zwischen ehrbaren Kaufleuten geübt zu werden pflegen, den kaufmännischen Einkommensbegriff beeinflussen.

Wie Wisselink in seinem Beitrag mit Recht betont hat, ist bei der wissenschaftlichen Untersuchung einer bestimmten Erscheinung zu unterscheiden zwischen der Erscheinung selbst, die den Gegenstand der Untersuchung bildet, und dem besonderen Zweck, dem die Untersuchung dient. Je nach dem Zweck der Untersuchung erscheint ein- und dieselbe Erscheinung in einem verschiedenen Lichte. Es ist hiernach nicht verwunderlich, daß Wirtschaftler, Wirtschaftsprüfer und Steuerexperten, die sich aus sehr verschiedenen Zweckrichtungen mit dem Phänomen des kaufmännischen Einkommens auseinanderzusetzen haben, zu verschiedenen Ergebnissen gelangen. Clements hat die unterschiedliche Position, die diese drei Expertengruppen zum kaufmännischen Einkommen einnehmen, in einem sehr anschaulichen Gedankenbild mit einem Dreieck verglichen, an dessen Ecken je einer der drei Experten steht, wobei die Mitte des Dreiecks die gemeinsame Basis bildet, von der aus jeder dieser drei Experten seine arteigene zweckbestimmte Konzeption entwickelt. Von diesem Gedankenbild auf die konkrete deutsche Situation überleitend, besteht zwischen dem Einkommensbegriff des Wirtschaftlers einerseits und den Einkommensbegriffen des Wirtschaftsprüfers und des Steuerexperten andererseits zunächst einmal in formaler Hinsicht ein bedeutsamer Unterschied: Der Einkommensbegriff des Wirtschaftsprüfers und der Einkommensbegriff des Steuerexperten sind in Deutschland weitgehend durch Gesetze rechtlich normiert, der des Wirtschaftsprüfers durch das Handelsrecht, der des Steuerexperten durch das Steuerrecht; dagegen entwickelt der Wirtschaftler seine Vorstellungen von Einkommen - frei von jeder rechtlichen Bindung - rein ratıonal

$\mathrm{m} \mathrm{a} \mathrm{b}$ blz. 333 
aus den praktischen Bedürfnissen der Unternehmensführung. Damit ergibt sich aber zugleich, daß die Einkommensbegriffe des Wirtschaftsprüfers und des Steuerexperten stets nur nationale Gültigkeit besitzen, daß indessen die Vorstellungen des Wirtschaftlers von Einkommen in allen Ländern mehr oder weniger miteinander übereinstimmen werden, weil die Bedürfnisse der Unternehmensführung sich in allen Ländern gleichen und demgemäß auch bei den Wirtschaftlern in allen Ländern die gleichen Vorstellungen von dem - in ihrem Sinne - ,richtigen” Einkommensbegriff wachrufen werden. Die internationale Gültigkeit des von den Wirtschaftlern als sachgerecht empfundenen Einkommensbegriffes tritt innerhalb des Beitrags von Clements deutlich zu Tage. Clements erblickt die Sonderheit, die den Einkommensbegriff des Wirtschaftlers von dem des Wirtschaftprüfers und dem des Steuerexperten unterscheidet, vor allem darin, daß der Wirtschaftler einmal eine Einkommensermittlung auf der Grundlage historischer Kosten ablehnt und statt dessen eine am Marktpreis orientierte Bewertung fordert und daß er ferner im Hinblick auf die sich beim Geldwert weltweit bemerkbar machenden inflationären Tendenzen anstelle einer reinen Geldwertrechnung eine Einkommensermittlung fordert, die dem Gesichtspunkt der gütermäßigen Substanzerhaltung hinreichend Rechnung trägt. Es bedarf kaum noch einer besonderen Erwähnung, daß die gleiche Forderung in allen Ländern erhoben wird, in denen sich inflationäre Tendenzen bemerkbar machen, aber der Wirtschaftsprüfer und der Steuerexperte dessenungeachtet auf der reinen Geldwertrechnung beharren oder beharren müssen, weil die für sie verbindlichen Gesetzesnormen sie dazu zwingen.

So wie hiernach die Dinge liegen, will mir scheinen, daß es nicht mehr erforderlich ist, den Einkommensbegriff des Wirtschaftlers, den Clements bereits so ausgezeichnet herausgearbeitet hat, in diesem Beitrag noch einmal zu behandeln. Ich glaube mich vielmehr darauf beschränken zu können, den handelsrechtlichen Einkommensbegriff, wie er in Deutschland von dem Wirtschaftsprüfer, und den steuerrechtlichen Einkommensbegriff, wie er von den deutschen Steuerexperten zu beachten ist, in kurzen Zügen darzustellen.

\section{Der handelsrechtliche und steuerrechtliche Vermögensvergleich}

Das von dem Kaufmann erwirtschaftete positive Einkommen wird im deutschen Handels- und Steuerrecht übereinstimmend als der "Gewinn" bezeichnet; ist es negativ, so handelt es sich um einen "Verlust”. Die Gewinnermittlung erfolgt handelsrechtlich und steuerrechtlich im Wege des Vermögensvergleichs dergestalt, daß das Geschäftsvermögen am Schluß des Wirtschaftsjahres - nach Ausschaltung der Privatentnahmen und -einlagen (bei Kapitalgesellschaften nach Ausschaltung der Gewinnausschüttungen und Kapitalerhöhungen) - mit dem Geschäftsvermögen am Anfang des Wirtschaftsjahres verglichen wird. Um den in dieser Weise vorgeschriebenen Vermögensvergleich sicherzustellen, bestimmt das Handelsgesetzbuch, daß der Kaufmann ,Bücher zu führen und in diesen seine Handelsgeschäfte und die Lage seines Vermögens nach den Grundsätzen ordnungsmäßiger Buchführung ersichtlich zu machen hat". Zum Schluß eines jeden Geschäftsjahres hat der Kaufmann zunächst ein „Inventar” aufzustellen, d.h. grundsätzlich im Wege einer körperlichen Bestandsaufnahme die ihm gehörenden Vermögensgegenstände festzustellen; das Gesetz verzichtet auf eine körperliche Bestandsaufnahme nur 
dann, wenn aufgrund eines den Grundsätzen ordnungsmäßiger Buchführung entsprechenden Verfahrens (z.B. aufgrund einer geeigneten Lagerbuchführung) die am Schluß des Geschäftsjahres vorhandenen Vermögensgegenstände nach Art, Menge und Wert in anderer Weise festgestellt werden können. Auf der Grundlage des so errichteten Inventars hat der Kaufmann eine Bilanz aufzustellen, in der er die einzelnen Vermögensgegenstände zu bewerten und die diesen Gegenständen gegenüberstehenden Schulden anzugeben hat. Diese ihm handelsrechtlich auferlegten Buchführungs- und Bilanzierungspflichten hat der Kaufmann zugleich im Interesse der Besteuerung zu erfüllen.

\section{Der Maßgeblichkeitsgrundsatz}

Die hier kurz skizzierten Buchführungs- und Bilanzierungspflichten besagen noch nichts über den materiellen Gehalt des handels- und steuerrechtlichen Gewinnbegriffes. Sie bilden allein den äußeren Rahmen, innerhalb dessen der Gewinn zu fixieren ist. Sein materieller Gehalt bestimmt sich hingegen nach den Bewertungsregeln, die im Handelsrecht für die Zwecke des handelsrechtlichen Vermögensvergleiches und - teilweise davon abweichend - im Steuerrecht für die Zwecke des steuerrechtlichen Vermögensvergleiches enthalten sind. Die nach den Bewertungsregeln des Handelsrechts entwickelte Handelsbilanz und die nach den Bewertungsregeln des Steuerrechts erstellte Steuerbilanz stehen im deutschen Recht - anders als z.B. im niederländischen Recht - nicht beziehungslos nebeneinander. Die Handelsbilanz bildet vielmehr kraft des im deutschen Steuerrecht geltenden Grundsatzes von der „Maßgeblichkeit der Handelsbilanz” auch die Grundlage der Besteuerung; die Steuerbehörde darf von den in der Handelsbilanz zulässigerweise gewählten Wertansätzen nur abweichen, sofern der Wertansatz in der Handelsbilanz gegen eine zwingende Bewertungsvorschrift des Steuerrechts verstößt. Solche steuerrechtlich bedingten Bewertungskollisionen sind allerdings nicht selten, weil das Steuerrecht durchweg eine bestimmte Mindestbewertung vorschreibt, während das Handelsrecht - abgesehen von dem am 1.1.1966 in Kraft getretenen neuen Aktiengesetz, das eine neue Lage geschaffen hat und auf das ich noch an anderer Stelle zurückkommen werde - nur Höchstwertvorschriften kennt und somit Unterbewertungen nicht verbietet. Der Grund für die hier zu Tage tretenden gegensätzlichen Bewertungstendenzen liegt auf der Hand: Das Handelsrecht will den Gläubiger schützen, der zur Beurteilung der Kreditwürdigkeit seines Schuldners in dessen Handelsbilanz Einsicht nimmt; aus dieser Zweckrichtung heraus hat das Handelsrecht keine Veranlassung, Unterbewertungen zu verbieten. Das Steuerrecht will dagegen aus Gründen der Besteuerungsgleichmäßigkeit eine objektive Gewinnermittlung gewährleisten; es ist aus diesem Grunde gezwungen, der Bewertung nach unten Grenzen zu setzen.

Abschließend sei noch auf eine beachtenswerte Sonderheit des deutschen Bilanzsteuerrechts hingewiesen, die sich aber zwingend aus dem Grundsatz von der steuerrechtlichen Maßgeblichkeit der Handelsbilanz ergibt. Da die Steuerbilanz stets aus der Handelsbilanz abgeleitet wird, so folgt, daß der Kaufmann in Fällen, in denen er handelsrechtlich ein Bewertungswahlrecht besitzt, einen niedrigeren Wert mit Wirkung für die Besteuerung nur dann geltend machen kann, wenn er den niedrigeren Wert auch in seiner Handelsbilanz eingesetzt hat. Hat z.B. der Kaufmann vor Jahren ein Grundstück für 100000 DM gekauft und ist er der 
Meinung, daß dieses Grundstück jetzt nur noch einen Wert von 60000 DM besitzt, so kann der Wert von $60000 \mathrm{DM}$ innerhalb der Steuerbilanz nur dann anerkannt werden, wenn der Kaufmann zuvor das Grundstück in seiner Handelsbilanz mit 60000 DM angesetzt hat. Ein anderes Beispiel: Berechnet der Kaufmann die Abschreibungen auf eine Maschine nach einer 10 jährigen Nutzungsdauer, so kann er die Abschreibungen für die Zwecke der Besteuerung nicht nach einer fünfjährigen Nutzungsdauer berechnen; in gleicher Weise kann er, wenn das Steuerrecht eine entgegenkommende Sonderabschreibung vorsieht, eine solche Sonderabschreibung mit steuerlicher Wirkung nur dann in Anspruch nehmen, wenn er die Sonderabschreibung auch in seiner Handelsbilanz vornimmt. Ein letztes Beispiel: Der Kaufmann kann für die Alterspensionen, die er seinen Arbeitnehmern zusagt, handelsrechtlich und steuerrechtlich Rückstellungen bilden. Da jedoch der Kaufmann nach deutscher Auffassung handelsrechtlich nicht verpflichtet ist, solche Rückstellungen zu bilden, so kann er die Pensionsrückstellungen mit steuerlicher Wirkung nur dann geltend machen, wenn er die Rückstellungen zunächst in seiner Handelsbilanz gebildet hat.

\section{Der Maßgeblichkeitsgrundsatz und die Grundsätze ordnungsmäßiger Buch- führung}

Der Kaufmann hat bei der Aufstellung seiner Handelsbilanz, insbesondere bei der Bewertung der in der Bilanz verzeichneten Vermögensgegenstände die dafür geltenden gesetzlichen Vorschriften und die Grundsätze zu beachten, die sich nach „gutem Kaufmannsbrauch" dafür herausgebildet haben; diese kaufmännischen Grundsätze werden im deutschen Handelsrecht als die „Grundsätze ordnungsmäßiger Buchführung" bezeichnet. Es entspricht z.B. altüberkomnener kaufmännischer Ubung, daß die in der Handelsbilanz zu verzeichnenden Vermögensgegenstände höchstens mit ihren - historischen - Anschaffungskosten bewertet werden dürfen; deshalb würde eine Bilanz, in der der Kaufmann seine Wertpapiere mit dem höheren Tageswert ansetzte, nach deutscher Auffassung nicht den Grundsätzen ordnungsmäßiger Buchführung entsprechen. Auf Grund dieser Betrachtungsweise geht die deutsche Rechtspraxis davon aus, daß die Höchstwerte, die das deutsche Aktienrecht den Aktiengesellschaften vorschreibt, als der kodifizierte Ausdruck eines Grundsatzes ordnungsmäßiger Buchführung nicht nur für die Aktiengesellschaften, sondern auch für alle anderen Unternehmensformen gelten. Ein anderes Beispiel: Es entspricht altüberkommener kaufmännischer Ubung, daß der Kaufmann ,schwebende Verträge”, d.h. Verträge, die von beiden Seiten noch nicht erfüllt sind, noch nicht bilanziert. Wird jedoch durch Preisveränderungen das Gleichgewicht von Leistung und Gegenleistung gestört, so entspricht es dem Prinzip der kaufmännischen Vorsicht, daß der Vertragspartner, der durch die Preisveränderung voraussichtlich eine Einbuße erleiden wird, für diese Einbuße eine Rückstellung bildet; eine Handelsbilanz, die diesem Risiko nicht Rechnung tragen würde, entspräche nicht den Grundsätzen ordnungsmäßiger Buchführung. Ein letztes Beispiel: Immaterielle Werte (z.B. ein Geschäftswert) dürfen nach überkommener kaufmännischer Ubung nur dann bilanziert werden, wenn sie käuflich erworben sind; eine Bilanz, in der der Kaufmann den von ihm selbst geschaffenen Geschäftswert auswiese, wäre insoweit mit den Grundsätzen ordnungsmäßiger Buchführung nicht zu vereinbaren. 
Die hier veranschaulichten Grundsätze ordnungsmäßiger Buchführung, die ihre Existenz allein aus dem Kaufmannsbrauch herleiten, sind nach deutscher Auffassung - in gleicher Weise wie die gesetzlich verankerten Bilanzierungsregeln - objektives Recht; sie können deshalb nicht im Wege der privatrechtlichen Vereinbarung abbedungen werden.

Die handelsrechtlichen Grundsätze ordnungsmäßiger Buchführung haben aber nicht nur Bedeutung für die Handelsbilanz. Da nach deutschem Steuerrecht, wie bereits erwähnt, kraft des Maßgeblichkeitsgrundsatzes die Handelsbilanz im Prinzip auch der Besteuerung zugrunde zu legen ist, erlangen die handelsrechtlichen Grundsätze ordnungsmäßiger Buchführung auch Wirksamkeit für das Steuerrecht; die nach den Grundsätzen ordnungsmäßiger Buchführung erstellte Handelsbilanz ist auch der Besteuerung zugrunde zu legen, es sei denn, das Steuerrecht bestimmte für diesen oder jenen Posten Gegenteiliges. Eine solche Divergenz zwischen Handels- und Steuerrecht ist z.B. beim käuflich erworbenen Geschäftswert zu verzeichnen. Obwohl die Grundsätze ordnungsmäßiger Buchführung eine angemessene Abschreibung der Geschäftswerte verlangen, ist steuerrechtlich eine solche Abschreibung nur dann zulässig, wenn der erworbene Geschäftswert nachweislich im Wert gesunken ist. Fehlt es aber an einer solchen steuerrechtlichen Sondernorm, hat die Steuerbehörde die Handelsbilanz unverändert hinzunehmen; es ist ihr insbesondere versagt, in der Steuerbilanz „Wirtschaftsgüter” anzusetzen, deren Ansatz in der Handelsbilanz nach den Grundsätzen ordnungsmäßiger Buchführung verboten ist, oder in der Steuerbilanz Passivposten zu streichen, deren Ansatz in der Handelsbilanz zwingend geboten ist. Du Steuerbehörden vertreten allerdings oft einen abweichenden Standpunkt.

\section{Privatrechtliche Bilanzierungsabreden und ihre Bedeutung für die Handels- und Steuerbilanz}

Wisselink vertritt in seinem Beitrag die Auffassung, daß für die Beantwortung der Frage, ob die Bilanz einer Gesellschaft richtig ist oder nicht, in erster Linie die zwischen den Gesellschaftern getroffenen Bilanzierungsabreden, insbesondere also der zwischen ihnen geschlossene Gesellschaftsvertrag, maßgeblich ist. Es muß leider festgestellt werden, daß die Auffassung Wisselinks mit dem deutschen Handels- und Steuerrecht nicht im Einklang steht. Die deutsche Rechtspraxis erblickt in den Bewertungsregeln des Handelsrechts und in den Grundsätzen ordnungsmäßiger Buchführung objektive Rechtsnormen mit öffentlich-rechtlichem Einschlag, die durch private Parteiabreden nicht abbedungen werden können. Es steht den Beteiligten selbstverständlich frei, z.B. für die Zwecke der Gewinnausschüttung eine ihren Abmachungen entsprechende "Privatbilanz" aufzustellen; die Erstellung einer solchen Privatbilanz entbindet sie aber nicht von der zwingenden Verpflichtung, außer einer solchen Privatbilanz noch eine Bilanz zu erstellen, die den handelsrechtlichen Bilanzierungsvorschriften und den Grundsätzen ordnungsmäßiger Buchführung entspricht, wobei für die Zwecke der Besteuerung nur die letztere Bilanz verbindlich ist. Diese Grundsätze des deutschen Rechts zielen darauf $a b$, der Bilanz eine objektive Aussagefähigkeit zu verleihen. Die Bedeutung, die diesem Umstand im Wirtschaftsleben zukommt, wird durch das neue am 1. 1.1966 in Kraft getretene Aktiengesetz unterstrichen, das den Aktiengesellschaften nicht nur eine Überschreitung, sondern - erstmals im deutschen Han- 
delsrecht - auch eine Unterschreitung der in dem neuen Gesetz vorgeschriebenen Werte verbietet. Es liegt auf der Hand, daß die gesetzlich festgelegte Mindestbewertung, durch die der Offentlichkeit ein Einblick in die wahre Ertragslage der Gesellschaft ermöglicht und den Minderheitsaktionären eine dieser Ertragslage entsprechende Dividendenzahlung gewährleistet werden soll, nicht durch private gesellschaftsrechtliche Abreden abbedungen werden kann. Es wird bereits - wenn auch vereinzelt - die Auffassung vertreten, daß die neuen aktienrechtlichen Bewertungsvorschriften nicht nur, soweit sie wie bisher eine Uberbewertung verbieten, sondern auch soweit sie eine bestimmte Mindestbewertung vorschreiben, nur die Kodifizierung einer ohnehin bestehenden kaufmännischen Übung bedeuten und daß deshalb die aktienrechtlich vorgeschriebene Mindestbewertung nicht nur für die Aktiengesellschaft, sondern auch für alle anderen Unternehmensformen (z.B. Einzelkaufmann, Personengesellschaft, $\mathrm{GmbH}$ ) Gültigkeit hätte. Die zurzeit noch herrschende Meinung geht nicht so weit, sie nimmt vielmehr an, daß die aktienrechtlich vorgeschriebene Mindestbewertung vorerst nur für die Aktiengesellschaft gilt; sie weist aber zugleich auf die Möglichkeit hin, daß im Laufe der Zeit die aktienrechtlich vorgeschriebene Mindestbewertung im Wege kaufmännischer Übung auch für die anderen Unternehmensformen Bedeutung erlangen könnte.

Die hier aufgezeigten Gesichtspunkte zeigen, daß die Lehre Wisselinks von dem Primat der privatrechtlichen Bilanzierungsvereinbarungen mit dem deutschen Handelsrecht nicht zu vereinbaren ist. Das deutsche Handelsrecht und - kraft des Maßgeblichkeitsgrundsatzes ihm folgend - auch das deutsche Steuerrecht erkennen nur solche Handelsbilanzen als ordnungsmäßig an, die den handelsrechtlichen Bewertungsregeln und den Grundsätzen ordnungsmäßiger Buchführung Rechnung tragen. Zudem kann nach allgemeiner Anschauung eine für die Offentlichkeit oder für öffentliche Zwecke bestimmte Bilanz nur dann einen Aussagewert haben, wenn sie einen Vergleich mit anderen Bilanzen gestattet. Dieser - im Wirtschaftsleben unerläßliche - Vergleich setzt jedoch voraus, daß die Vermögensgegenstände objektiv bewertet worden sind.

\section{Besonderer Teil}

\section{Vorbemerkung}

In den nachfolgenden Abschnitten werden die für die einzelnen Bilanzpositionen geltenden aktienrechtlichen und steuerrechtlichen Bewertungsregeln einander gegenübergestellt. Die aktienrechtlichen Bewertungsvorschriften legen die Aktiengesellschaft auf eine bestimmt geartete Bewertung fest, die zwar den Gesellschaften einen gewissen Bewertungsspielraum beläßt, die aber im Grundsatz weder überschritten noch unterschritten werden darf; für die Nicht-Aktiengesellschaften (Einzelkaufmann, Personengesellschaften und $\mathrm{GmbH}$ ) haben die aktienrechtlichen Bewertungsregeln nur den Charakter von Höchstwertvorschriften, von denen nicht nach oben, wohl aber nach unten abgewichen werden darf. Soweit ein handelsrechtlich zulässiger Wert unter dem steuerlich zulässigen Mindestwert liegt, ist in der Steuerbilanz - abweichend von der Handelsbilanz - der steuerliche Mindestwert einzusetzen. 


\section{Die Bewertung des Anlagevermögens}

a) Aktienrechtliche Bewertung

Gegenstände des Anlagevermögens sind mit den Anschaffungs- oder Herstellungskosten, vermindert um die Abschreibungen, anzusetzen. Bei der Berechnung der Herstellungskosten - insbesondere bei solchen von selbst hergestellten Anlagegütern - dürfen in angemessenem Umfang Abnutzungen sowie angemessene Teile der Betriebs- und Verwaltungskosten eingerechnet werden.

Die Abschreibungen sind bei Gegenständen, die der Absetzung unterliegen, planmäßig vorzunehmen. Der Plan muß die Anschaffungs- oder Herstellungskosten nach einer den Grundsätzen ordnungsmäßiger Buchführung entsprechenden Abschreibungsmethode auf die Geschäftsjahre verteilen, in denen der Gegenstand voraussichtlich genutzt werden kann. Außerplanmäßige Abschreibungen sind zulässig, wenn der Gegenstand eine besondere Wertminderung erfahren hat oder wenn das Steuerrecht eine niedrigere Bewertung zuläßt.

\section{b) Steuerrechtliche Bewertung}

\section{aa) Bewegliche Gegenstände}

Die mit den Anschaffungs- oder Herstellungskosten anzusetzenden beweglichen Wirtschaftsgüter sind, verteilt auf die betriebsgewöhnliche Nutzungsdauer, entweder linear oder degressiv abzuschreiben. Wird eine degressive Abschreibungsmethode (z.B. die Abschreibung vom jeweiligen Restwert) gewählt, so darf der anzuwendende Hundertsatz das zweifache des linearen Abschreibungssatzes und 20 v.H. der Anschaffungs- oder Herstellungskosten nicht überschreiten. Soll z.B. eine Maschine mit einer betriebsgewöhnlichen Nutzungsdauer von 10 Jahren degressiv jeweils vom Restwert abgeschrieben werden, so darf somit der Abschreibungssatz 20 v.H. nicht übersteigen; der degressive Höchstabschreibungssatz von 20 v.H. der Anschaffungs- oder Herstellungskosten darf auch dann nicht überschritten werden, wenn die Nutzungsdauer fünf Jahre und weniger beträgt; es empfiehlt sich deshalb, bei beweglichen Wirtschaftsgütern mit einer Nutzungsdauer bis zu fünf Jahren stets die - in diesem Fall höhere - lineare Abschreibung zu wählen.

bb) Ge bä u de

Altgebäude - das sind Gebäude, die bis zum Jahre 1924 erstellt worden sind - sind jährlich mit $2^{1 / 2}$ v.H., neuere Gebäude mit jährlich 2 v.H. der Anschaffungsoder Herstellungskosten linear abzuschreiben. Kann davon ausgegangen werden, daß bei den Altgebäuden die tatsächliche Nutzungsfähigkeit weniger als 40 Jahre und bei den neueren Gebäuden weniger als 50 Jahre betragen wird, so kann ein entsprechend höherer Abschreibungssatz gewählt werden. Besonders begünstigt sind Gebäude, die ab dem Jahre 1965 neu erstellt worden sind; diese Gebäude können degressiv abgeschrieben werden, und zwar dergestalt, daß der Abschreibungssatz in den ersten 12 Jahren 3,5 v.H., in den darauffolgenden 20 Jahren 2 v.H. und in den letzten 18 Jahren 1 v.H. der Herstellungskosten beträgt. Das Recht zur degressiven Abschreibung steht nur dem Ersteller des Gebäudes (dem Bauherrn) zu; käuflich erworbene Gebäude können nur linear abgeschrieben werden. 


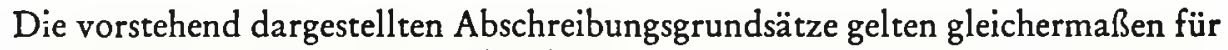
Wohngebäude wie auch für Betriebsgebäude.

\section{cc) Sonderabschreibungen}

Steuerliche Investitionsanreize dergestalt, daß - nach Art der englischen Investment-Allowance - eine die Anschaffungs- oder Herstellungskosten übersteigende „Abschreibung" gewährt wird, sind dem deutschen Steuerrecht fremd. Volkswirtschaftlich wünschenswerte Investitionen werden durch Sonderabschreibungen gefördert, wobei im Jahr der Anschaffung oder Herstellung neben der Normalabschreibung bei Gebäuden die Sonderabschreibung von insgesamt 30 v.H. und bei beweglichen Wirtschaftsgütern eine solche von 50 v.H. vorgenommen werden darf. In dieser Weise sind insbesondere begünstigt:

Investitionen in Krankenanstalten,

Investitionen zur Verhinderung von Schädigungen durch Abwässer,

Investitionen zur Reinerhaltung der Luft und zur Lärmbekämpfung, gewisse Investitionen im Bergbau.

Für in Berlin (West) ansässige Unternehmen gelten besondere Abschreibungsvergünstigungen, die im Anschaffungs- oder Herstellungsjahr Abschreibungen bis zur Höhe von 75 v.H. der Anschaffungs- oder Herstellungskosten zulassen.

\section{Umlaufvermögen}

a) Aktienrechtliche Bewertung

Die Gegenstände des Umlaufvermögens (z.B. Handelsware, Roh, Hilfs- und Betriebsstoffe, Halb- und Fertigfabrikate) sind grundsätzlich zu den Anschaffungsoder Herstellungskosten anzusetzen; liegt der Wert, der den Gegenständen am Abschlußstichtag beizulegen ist, insbesondere deren Börsen- oder Marktpreis, unter den Anschaffungs- oder Herstellungskosten, so ist dieser niedrigere Wert anzusetzen (sog. Niederstwertprinzip). Es ist zulässig, die Gegenstände mit einem Wert anzusetzen, der noch unter dem Börsen- oder Marktpreis liegt, soweit der niedrigere Wertansatz bei vernünftiger kaufmännischer Beurteilung notwendig ist, um zu verhindern, daß in der nächsten Zukunft der Wertansatz dieser Gegenstände auf Grund von Wertschwankungen geändert werden muß; ein niedrigerer Wertansatz darf auch gewählt werden, wenn der gewählte Wert für die Zwecke der Besteuerung für zulässig gehalten wird.

Die Herstellungskosten der Halb- und Fertigfabrikate sind mindestens mit den Materialkosten und den aufgewandten Fertigungslöhnen anzusetzen; es dürfen jedoch in angemessenem Umfang auch die Abnutzung und angemessene Teile der Betriebs- und Verwaltungskosten (z.B. die Material- und Fertigungsgemeinkosten) in die Herstellungskosten einbezogen werden.

Soweit es den Grundsätzen ordnungsmäßiger Buchführung entspricht, dürfen die Gegenstände des Vorratsvermögens auch nach der Lifo- oder Fifo-Methode bewertet werden.

b) Steuerrechtliche Bewertung

Die steuerrechtlichen Bewertungsgrundsätze decken sich im Grundsatz mit denen des Aktienrechts; jedoch sind die folgenden Abweichungen zu beachten: 
Eine Bewertung mit einem unter dem Börsen- oder Marktpreis liegenden Wert ist im Grundsatz unzulässig; jedoch kann bei Waren, die starken Preisschwankungen unterliegen, die Preisentwicklung, die sich 4 bis 6 Wochen vor und nach dem Bilanzstichtag vollzieht, berücksichtigt werden. - Bestimmte aus dem Ausland bezogene Rohstoffe dürfen generell mit einem Abschlag von 20 v.H. angesetzt werden; besonders begünstigt ist bei gewissen aus dem Ausland bezogenen Rohstoffen der Mehrbestand, für den ein Abschlag von 30 v.H. gewährt wird. - Hat sich der Börsen- oder Marktpreis einer Ware oder eines Fabrikates im Laufe des Wirtschaftsjahres um mehr als 10 v.H. erhöht, so darf eine Rücklage für Preissteigerung gebildet werden, die jedoch spätestens am Ende des 6. Jahres wieder zugunsten des Ergebnisses aufzulössen ist.

In den Herstellungskosten sind außer den Materialkosten und Fertigungslöhnen stets auch die Material- und Fertigungsgemeinkosten einzubeziehen. $\mathrm{Zu}$ den Herstellungskosten gehört auch der Wertverzehr des Anlagevermögens, soweit es der Fertigung der Erzeugnisse gedient hat.

Die Bewertung nach der Lifo-Methode ist nur zulässig, wenn der Kaufmann tatsächlich die zuletzt erworbene Ware stets zuerst veräußert; ist das nichts feststellbar, so erfolgt eine Durchschnittsbewertung nach dem gewogenen Mittel der im Laufe des Wirtschaftsjahres erworbenen und der ggf. zu Beginn des Wirtschaftsjahres vorhandenen Gegenstände.

\section{Forderungen}

Forderungen sind aktienrechtlich und steuerrechtlich mit dem Wert anzusetzen, der ihnen am Abschlußstichtag beizulegen ist. Diesem Grundsätz gemäß sind nicht nur die uneinbringlichen Forderungen auszubuchen, es ist vielmehr in der Form des Delkredere auch das latente Risiko zu berücksichtigen, mit dem das Unternehmen erfahrungsgemäß zu rechnen hat; dieses Risiko wird regelmäßig an den Ausfällen gemessen, die das Unternehmen in der Vergangenheit tatsächlich erlitten hat. In das Delkredere sind auch die Finanzierungskosten, die der Forderungsbestand verursache, sowie die Kosten zu berücksichtigen, die der Einzug der Forderungen mit sich bringt. Unverzinsliche Forderungen sind abzuzinsen.

\section{Verbindlichkeiten}

Verbindlichkeiten sind aktienrechtlich und steuerrechtlich mit ihrem Rückzahlungsbetrag anzusetzen. Ist der Rückzahlungsbetrag höher als der Ausgabebetrag, so ist der Kaufmann handelsrechtlich berechtigt und steuerrechtlich verpflichtet, den Unterschiedsbetrag als Aktivum abzusetzen (sog. Damnum). Das Damnum ist planmäßig abzuschreiben.

\section{Rückstellungen}

a) Aktienrechtliche Zulässigkeit und Bewertung

Rückstellungen dürfen nur gebildet werden

aa) für ungewisse Verbindlichkeiten (z.B. für Prozeßrisiken)

bb) für drohende Verluste aus schwebenden Geschäften (z.B. wenn die auf Termin gekauf te Ware inzwischen im Preise gefallen ist), 
cc) für im Geschäftsjahr unterbliebene Instandhaltung, aber nur unter der Voraussetzung, daß die Instandhaltung im folgenden Geschäftsjahr nachgeholt wird,

dd) für Gewährleistungen, die ohne rechtliche Verpflichtungen erbracht werden (z.B. die Nachbesserung und der Kundendienst für bereits ausgelieferte Fabrikate),

ee) für laufende Pensionszahlungen und für die Anwartschaften auf künftige Pensionszahlung.

Für andere Zwecke, z.B. für die Zwecke der Substanzerhaltung, dürfen Rückstellungen nicht gebildet werden.

Der Höhe nach sind die Rückstellungen stets auf das Maß zu beschränken, das nach vernünftiger kaufmännischer Beurteilung notwendig ist.

b) Steuerrechtliche Zulässigkeit und Bewertung

In den Fällen, in denen das Aktienrecht die Bildung einer Rückstellung für zulässig erklärt, ist eine Rückstellungsbildung auch steuerrechtlich zulässig. Über das Maß des Notwendigen werden allerdings Kaufmann und Steuerbehörde nicht selten sehr verschiedener Meinung sein.

\section{Veräußerungsgewinne}

Gewinne, die bei der Veräußerung von Gegenständen des Anlagevermögens erzielt werden, sind aktienrechtlich Bestandteil des Gewinns; steuerrechtlich wurden solche Gewinne ehedem - nicht anders wie die Umsatzgewinne - der vollen Besteuerung unterworfen. Seit dem Jahre 1965 ist es möglich, die Besteuerung bestimmt gearteter Veräußerungsgewinne in die Zukunft zu verlagern. Gewinne, die bei der Veräußerung von Grund und Boden, Gebäuden, Anteilen an Kapitalgesellschaften und gewissen anderen Gütern entstehen, dürfen, wenn sie innerhalb von zwei Jahren - bei der Neubeschaffung von Gebäuden innerhalb von vier Jahren - reinvestiert werden, von den Anschaffungskosten der neu beschafften Gegenstände abgezogen werden, wobei indessen die folgenden Reinvestitionsbeschränkungen zu beachten sind:

Uneingeschränkt können die vorstehend bezeichneten Veräußerungsgewinne auf die Anschaffungs- oder Herstellungskosten neubeschaffter Gebäude und neubeschaffter beweglicher abnutzbarer Güter übertragen werden. Eine Ubertragung auf die Anschaffungskosten von neu beschafftem Grund und Boden ist nur zulässig, wenn der zu übertragende Veräußerungsgewinn bei der Veräußerung von Grund und Boden entstanden ist. Eine Übertragung von Veräußerungsgewinnen auf neu-beschaffte Anteile an Kapitalgesellschaften ist gestattet, sofern der zu übertragende Veräußerungsgewinn bei der Veräußerung von Anteilen an Kapitalgesellschaften entstanden ist und das Bundesministerium für Wirtschaft die volkswirtschaftliche Förderungswürdigkeit des Vorgangs bescheinigt.

Die Neuregelung ist in ihren Einzelheiten außerordentlich kompliziert; sie kann hier nur in Umrissen beschrieben werden.

\section{Ausgaben}

Im Aktiengesetz sind besondere Vorschriften über die Behandlung des Ausgaben 
entbehrlich. Nach deutschem Steuerrecht sind alle Ausgaben abzugsfähig, die „durch den Betrieb veranlaßt sind” (sog. Betriebsausgaben). Nicht abzugsfähig sind

a) bei den natürlichen Personen:

aa) Privatentnahmen; Privatentnahmen sind alle Aufwendungen für die private Lebensführung einschließlich derjenigen, die die wirtschaftliche oder gesellschaftliche Stellung mit sich bringt; die letzteren sind auch dann nicht abzugsfähig, wenn sie gleichzeitig der Förderung des Berufes oder der gewerblichen Tätigkeit des Steuerpflichtigen dienen; die rein betrieblich veranlaßte Repräsentation ist jedoch abzugsfähig;

bb) Geschenke, die den Betrag von 100 DM je Empfänger und Geschäftsjahr überschreiten;

cc) Spenden für wohltätige und wissenschaftliche Zwecke, soweit sie bestimmte Grenzen überschreiten;

dd) Steuern vom Einkommen;

b) bei den juristischen Personen:

aa) die offenen und verdeckten Gewinnausschüttungen,

bb) die Aufwendungen für die Erfüllung satzungsmäßiger Zwecke,

cc) Vergütungen, die den Mitgliedern des Aufsichtsrats gewährt werden,

dd) die zu Buchstabe a Doppelbuchstaben bb) bis dd) bezeichneten Ausgaben.

Die Vermögensteuer ist nur bei natürlichen Personen mit Wohnsitz im Inland abzugsfähig.

\section{Anbang}

\section{Die Körperschaftsteuer und das Schachtelprivileg}

Die deutsche Körperschaftsteuer hat seit eh und je den Charakter einer voll ausgebildeten zweiten Einkommensteuer: Der Gewinn der Kapitalgesellschaft unterliegt zunächst der Körperschaftsteuer, der ausgeschüttete Gewinn (Dividende) wird beim empfangenden Gesellschafter (Aktionär) noch einmal der Einkommensteuer unterworfen. Der Steuersatz der Körperschaftsteuer beträgt 51 v.H. für den nicht ausgeschütteten Gewinn (thesaurierter Gewinn zuzüglich der nicht abzugsfähigen Ausgaben) und 15 v.H. für den ausgeschürteten Gewinn. Bei den sog. personenbezogenen Kapitalgesellschaften - das sind Kapital-gesellschaften, deren Vermögen 5 Millionen DM nicht übersteigt und deren Anteile sich mindestens zu 76 v.H. in der Hand von natürlichen Personen befinden - betragen die entsprechenden Steuersätze 49 v.H. und 26,5 v.H. Ausländische Kapitalgesellschaften unterliegen mit ihren inländischen Gewinnen der deutschen $\mathrm{KSt}$ zu einem Steuersatz von 49 v.H.

Dividenden und sonstige Gewinnausschüttungen, die eine Kapitalgesellschaft als Gesellschafterin einer anderen Kapitalgesellschaft vereinnahmt, unterliegen der vollen Körperschaftsteuer. Ist jedoch die empfangende Gesellschaft (Obergesellschaft) mindestens zu einem Viertel an der ausschüttenden Gesellschaft (Untergesellschaft) beteiligt, so bleibt die empfangene Dividende steuerfrei (sog. Schachtelprivileg). Der Obergesellschaft wird aber in diesem Fall eine Sondersteuer 
auferlegt, deren Steuersatz 36 v.H. der steuerfrei empfangenen Dividende beträgt. Diese Steuer wird erhoben, weil der Untergesellschaft für ihre Ausschüttung an die Obergesellschaft der ermäßigte Ausschüttungssteuersatz gewährt wird, dieser Steuersatz aber materiell nur dann gerechtfertigt erscheint, wenn die Dividende an natürliche Personen gezahlt wird. Da diese Voraussetzung bei der Ausschüttung an die Obergesellschaft nicht gegeben ist, hat diese die erwähnte Sondersteuer in Höhe des Unterschiedes zwischen dem Normalsteuersatz (51 v.H.) und des Ausschüttungssteuersatzes (15 v.H.) "nachzuzahlen”. Die so motivierte Sondersteuer bedeutet für die Obergesellschaft eine echte steuerliche Belastung nur dann, wenn sie die empfangene Dividende thesauriert. Verwendet sie indessen die empfangene Dividende zu einer erhöhten Dividendenzahlung, so wird der Nachteil der Nachsteuer durch die Gewährung des ermäßigten Steuersatzes für die erhöhte Dividende kompensiert. Úbersteigt die von der Obergesellschaft gezahlte Dividende deren steuerpflichtigen Gewinn - diese Voraussetzung ist oft bei Holdings gegeben - so wird die Nachsteuer insoweit nicht erhoben.

Das vorstehend gekennzeichnete Schachtelprivileg wird nur gewährt, wenn Obergesellschaft und Untergesellschaft in Deutsland unbeschränkt steuerpflichtig sind. In einigen Doppelbesteuerungsabkommen ist vorgesehen, daß das Schachtelprivileg auch für die Beteiligung an einer ausländischen Gesellschaft gewährt wird.

\section{Organschaft}

Grundsätzlich hat jede Person - mag es sich um eine natürliche oder eine juristische Person handeln - den von ihr erwirtschafteten Gewinn selbst zu versteuern. Eine Sonderheit gilt im deutschen Steuerrecht für Kapitalgesellschaften (Organgesellschaften), die mindestens zu 51 v.H. von einem anderen Unternehmen (Organträger) beherrscht werden und die wirtschaftlich und organisatorisch in das Unternehmen des Organträgers eingegliedert sind. Wird in einem solchen Falle zwischen dem Organträger und der Organgesellschaft ein Ergebnisabführungsvertrag abgeschlossen, kraft dessen sich die Organgesellschaft verpflichtet, ihren Gewinn an den Organträger abzuführen und in dem der Organträger sich verpflichtet, etwaige Verluste der Organgesellschaft zu decken, so wird fortan der Gewinn der Organgesellschaft in der Person des Organträgers besteuert; hat die Organgesellschaft Verlust, so ist der Organträger berechtigt, den Verlust der Organgesellschaft gegen den eigenen Gewinn zu verrechnen. Der hier dargestellte Grundsatz erfährt dadurch eine Einschränkung, daß nach der höchstrichterlichen Rechtsprechung die Organgesellschaft die bei ihr angefallenen nicht abzugsfähigen Ausgaben stets als eigenes Einkommen zu versteuern hat.

Als Organträger kann sowohl eine Kapitalgesellschaft als auch ein Personenunternehmen auftreten. Ist Organträger eine Kapitalgesellschaft, so schaltet die Organschaft die zweite Körperschaftsteuer, die das Schachtelprivileg nur in begrenztem Umfang vermeidet, nahezu vollkomen aus. Ist Organträger ein Personenunternehmen, so reduziert die Organschaft die steuerliche Belastung des von der Organgesellschaft erzielten Gewinnes auf die einmalige Einkommensbesteuerung in der Person der Gesellschafter.

Die Einheit der Einkommensermittlung berührt nicht die im übrigen fortbestehende subjektive Steuerpflicht der Organgesellschaft. Alle Umsatzgeschäfte, die sich $\mathrm{zwischen}$ Organ und Organträger vollziehen, sind deshalb zu angemes- 
senen Preisen abzurechnen und führen zur vollen Besteuerung der so errechneten Gewinne.

Die körperschaftsteuerliche Organschaft ist nicht im Gesetz geregelt, sie wurde im Wege der wirtschaftlichen Betrachtungsweise von der Rechtsprechung entwickelt. Es bestehen zurzeit mannigfache Zweifelsfragen. Die Rechtsprechung zieht insbesondere neuestens in Zweifel, ob sie die Organschaft zu Personenunternehmen noch länger aufrecht erhalten kann. Es wird zurzeit geplant, die körperschaftsteuerliche Organschaft einer gesetzlichen Regelung zuzuführen. Eine solche Regelung ist auch deshalb dringend erwünscht, weil das Steuerrecht der Anpassung an das Konzernrecht bedarf, das auf Grund des neuen Aktiengesetzes eine grundlegende Neugestaltung erfahren hat. 